\title{
Glucose Metabolism Disorder
}

National Cancer Institute

\section{Source}

National Cancer Institute. Glucose Metabolism Disorder. NCI Thesaurus. Code C53655.

A metabolic disorder characterized by abnormal blood glucose levels. 